\title{
The Synthesis of Sparse Antenna Array Based on Improved Mind Evolution Algorithm
}

\author{
Hongliang Tian
}

Henan Zhumadian Power Supply Company, Zhumadian, China

\begin{abstract}
Mind Evolution Algorithm (MEA) imitates the human mind evolution by using similartaxis and dissimilation operations, which overcomes the prematurity and improves searching efficiency. But the generation of the initial population is blind and the addition of naturally washed out temporary subpopulations is random. This paper improved MEA by introducing chaos and difference into it and proposed Chaos Difference Mind Evolution Algorithm (CDMEA), which brought adequate diversity to the initial population and saved the excellent genes in the evolution. Then CDMEA is used in the synthesis of sparse antenna arrays. The excellent results confirmed the effectiveness, flexibility and suitability of CDMEA.
\end{abstract}

Index Terms: MEA; chaos; CDMEA; sparse antenna array; side lobe level

(C) 2011 Published by MECS Publisher. Selection and/or peer review under responsibility of the International Conference on E-Business System and Education Technology

\section{Introduction}

With the rapid development of the telecommunications industry, the electromagnetic environment of space is increasingly deteriorating and the quality of communication is declining. To solve these problems, smart antenna which has the ability of low side-lobes, strong directional and anti-interference has been a great deal of concern. The antenna pattern synthesis problem becomes a hot research. Antenna array synthesis means in a given antenna radiation pattern or antenna performance, design antenna array element number, element spacing, elements' current amplitude and phase distribution to obtain special radiation characteristic.

Mind Evolution Algorithm (MEA) is brought forward by Professor K. M. Xie based on the analysis of human mind development and imitation the similartaxis and dissimilation phenomena in human society [1]. But it also has some shortcomings. For instance, the generation of the initial population is blind and the addition of naturally washed out temporary subpopulations is random. This paper introduced chaos and difference into MEA and proposed Chaos Difference Evolution Algorithm (CDMEA), which brought adequate diversity to the initial population and saved the excellent genes in the evolution. Compared with MEA, CDMEA is more exactly and effectively.

* Corresponding author.

E-mail address: linanwd@163.com 


\section{Chaos Difference Mind Evolution Algorithm(CDMEA)}

\section{A. Mind Evolution Algorithm (MEA)}

MEA inherited the idea of "population" and "evolution" from GA, divided a population into two kinds of subpopulations, superior subpopulations and temporary subpopulations. Superior subpopulations note the winners' information in global, temporary subpopulations note the process of the global competitive. Firstly, MEA takes similartax operation on all the subpopulations and search optimal value in the local quickly by comparing fitness functions. Then dissimilation operation is used to search in the whole solution space, choice the better individuals as the centers and create new temporary subpopulations. The individuals in the subpopulations post their information on the local bulletin board. The global bulletin board is used to post each subpopulation's information.

\section{B. Chaos Mapping}

The chaos phenomenon is the common phenomenon in the nonlinear dynamic systems. The chaos's behaviors are complex and similar to the random process, but have the inherent property of regularity. The chaos optimization algorithm is sensitively to the initial value, easily to jump out the local minimum, and quickly to search out the global optimization. It has the property of global asymptotical convergence [2-4].

Tent mapping is an important model of Chaos Dynamics with uniform probability density, power spectral density and ideal related characteristics. It is can be expressed as follow:

$$
x_{k+1}=\left\{\begin{array}{l}
2 x_{k}, 0 \leq x_{k} \leq 0.5 \\
2\left(1-x_{k}\right), 0.5<x_{k}<1
\end{array}\right.
$$

Its probability density function is:

$$
\rho(x)=1
$$

Tent mapping has simple structure and good ergodic uniformity, more suitable for a large number of data processing sequences. But there are small iterative cycle and unstable periodic point in Tent mapping. It will make the iteration to the fixed point 0 . In order to avoid iterates to fixed point, this paper uses the following method to improve Tent mapping:

$$
x(k+1)= \begin{cases}x(k) / 0.4 & x(k) \leq 0.4 \\ (1-x(k)) / 0.6 & x(k)>0.4\end{cases}
$$

We can see that from Fig.1, the points generated by improved Tent mapping are scattered uniformly in [0.1]. It overcomes its own lacks, such as small cycle and instability cycle points. 


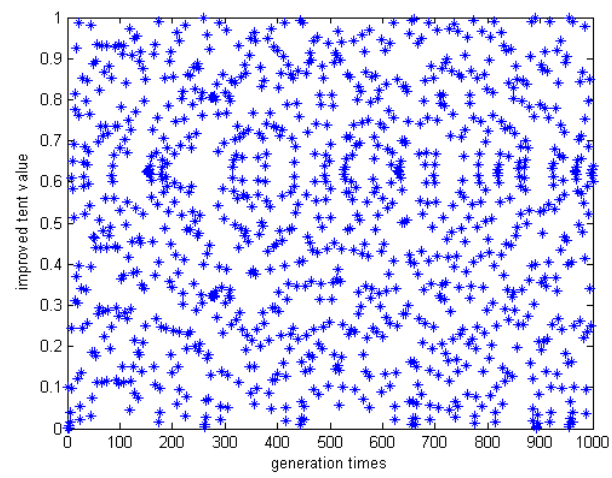

Figure 1. The chaos state of improved Tent mapping

\section{Difference Evolution Algorithm (DEA)}

DEA is proposed by Rainer Storn and Kennth Price according to the evolutionary law of nature [5]. It is a real coding algorithm with simple configuration, quick convergence rate and strong stability. There are three kinds of operations in DEA: mutation, crossover and selection operations. And mutation operation is the key operation in DEA. Its specific operation is: firstly select any two individuals in the population and compute their difference, then weight sum the difference and another individual to create new individual.

Let $X_{i, G}, i=1,2, \ldots, N$ is the individual in generation $G, G$ is the generation time, $N$ is the population size. The new individual can be created by following:

$V_{i, G+1}=d f\left(X_{r_{1}, G}-X_{r_{2}, G}\right)+X_{r_{3}, G}$

where $r_{1}, r_{2}$ and $r_{3}$ are three different integers selected randomly in $[1, N] . d f \in[0,2]$ is the mutation factor to control the scaling of $X_{r_{1}, G}-X_{r_{2}, G}$.

\section{Chaos Difference Mind Evolution Algorithm(CDMEA)}

MEA simulated the similartaxis and dissimilation phenomenon in human society and resolved the problem of prematurity and low convergence speed of traditional Intelligent Algorithm to a certain extent. But it also has some defects. For instance, the generation of the initial population is blind and the addition of naturally washed out temporary subpopulations is random. This paper introduced improved Tent mapping and the mutation operation of DEA into MEA and proposed CDMEA, which brought adequate diversity to the initial population and saved the excellent genes in the evolution.

The CDMEA is described as following:

Step1 Set evolutionary parameters: population size, subpopulation size and conditions for end;

Step2 Initialization: scatter individuals in terms of (3) in the whole solution space;

Step3 Similartax: individuals are produced by normal distribution with variance around each winner and the individual with highest score is the new winner replacing the old one in following steps;

Step4 Dissimilation: realize global optimization, some with lower score are washed out and replaced by new ones scattered according to (4) in the solution space;

Step5 Conditions for end: if the end conditions are filled, turn to step6; else repeat step3 and step 4;

Step6 Output evolutionary result, algorithm ends. 


\section{Antenna Array Synthesis}

Antenna array is a kind of antenna consisted of some array elements to obtain special radiation characteristic. According to the geometry structure, it can be divided into several categories: linear antenna array, rectangle antenna array and circular antenna array.

\section{E. Uniform Linear Antenna Array}

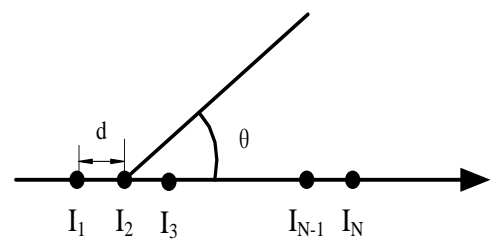

Figure 2. The model of uniform linear antenna

For a linear antenna array with given elements and spacing arranged in axis as it shows in Fig.2, its pattern formula is:

$F(\theta)=\sum_{n=1}^{N} I_{n} \exp \left[j(n-1) k d \cos \theta+\varphi_{n}\right]$

where $I_{n}$ is the $n$th element's amplitude; $\varphi_{n}$ is the $n$th element's phase; $\theta$ is the angle between the array axis and the ray; $d$ is the space between the adjacent elements; $k=2 \pi / \lambda$ is wave number.

Let the antenna array's main-lobe point at $\theta_{0}$, then $\varphi_{n}=-(n-1) k d \cos \theta_{0},(5)$ can be written as following:

$F(\theta)=\sum_{n=1}^{N} I_{n} \exp \left[j(n-1) k d\left(\cos \theta-\cos \theta_{0}\right)\right]$

\section{F. Uniform Circular Antenna Array}

Compared with linear antenna array and rectangle antenna array, uniform circular antenna array has bigger scanning range reached to $360^{\circ}$ and counteracting mutual coupling effect with the symmetric uniform distribution.

As Fig. 3 shows, there are $N$ elements distributing on a circle, $R$ is the circle's radius. $\theta \in[0, \pi / 2]$ is the angle between signal incident direction and axis $z, \phi \in[0,2 \pi]$ is the angle between the direction that the signal incident direction projected on the $x y$ plane. $\vec{r}=(\sin \theta \cos \phi, \sin \theta \sin \phi, \cos \theta)$ is the direction vector and $\vec{p}_{n}=\left(\cos \phi_{n}, \sin \phi_{n}, 0\right)$ is the position vector. $\phi_{n}=2 \pi n / N$ is the $n$th element's azimuth angle. 


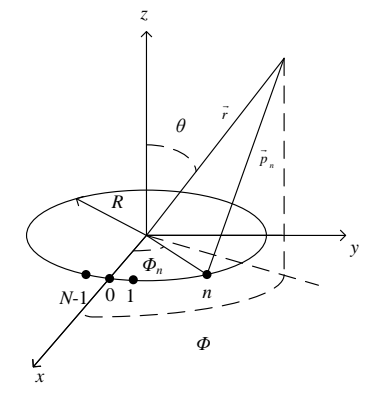

Figure 3. Uniform circular antenna array

The uniform circular antenna array's pattern function is:

$F(\theta, \phi)=\sum_{n=0}^{N-1} I_{n} e^{j\left[\beta_{n}-k R \cos \left(\phi-\phi_{n}\right) \sin \theta\right]}$

where $I_{n}$ is the $n$th element's amplitude and $\beta_{n}$ is the $n$th element's phase.

If let the antenna array's main lobe point at $\left(\theta_{0}, \phi_{0}\right)$, then $\beta_{n}=k R \cos \left(\phi_{0}-\phi_{n}\right) \sin \theta_{0}$, (7) can be written as following:

$F\left(\theta, \quad \phi ; \quad \theta_{0}, \quad \phi_{0}\right)=\sum_{n=0}^{N-1} I_{n} e^{j k R\left[\cos \left(\phi_{0}-\phi_{n}\right) \sin \theta_{0}-\cos \left(\phi-\phi_{n}\right) \sin \theta\right]}$

If only considering the antenna pattern on the $x y$ plane, then $\theta=\theta_{0}=90^{\circ}$, the pattern function can be written as following:

$F\left(\phi ; \quad \phi_{0}\right)=\sum_{n=0}^{N-1} I_{n} e^{j k R\left[\cos \left(\phi_{0}-\phi_{n}\right)-\cos \left(\phi-\phi_{n}\right)\right]}$

\section{G. Sparse Antenna Array}

In the engineering application of antenna array, narrow main lobe is often used to improve spatial resolution. To gain narrow main lobe in a full matrix antenna array, we have to enlarge the antenna array's aperture. But it not only adds the cost but also increases the complexity of the antenna system. Distributing elements sparsely is another method to gain main lobe which saved the cost, improved the spatial resolution and avoided grating lobe.

\section{Simulation results}

\section{H. Linear Sparse Antenna Array}

A linear sparse antenna array in fact is an unequal linear array with unequal space between the adjacent elements. Its pattern function can be written as following: 
$F(\theta)=\sum_{n=1}^{N} \exp \left(j k d_{n} \cos \theta\right)$

where $d_{n}$ is the space between the first element and the nth element.

Before optimize the linear sparse array, we can pre-treat the position variables to reduce the search range and improve the optimization efficiency [6].

Let the first element's position variable is $d_{1}=0, d_{c}$ is the position variable's difference between two adjacent elements:

$\min \left\{d_{i}-d_{j}\right\} \geq d_{c}, \quad 1 \leq j \leq i \leq N$

Then the left solution space is:

$L Z=L-2 d_{c}-d_{c}(N-3)=L-(N-1) d_{c}$

Scatter $N$-2 individuals composing initial population in the left solution space and sort them ascending to compose $X=\left[x_{1}, x_{2}, \cdots, x_{N-1}\right]^{T}$. Then the $N-2$ position variables in the linear sparse array can be written as:

$D=\left[\begin{array}{c}d_{2} \\ d_{3} \\ \vdots \\ d_{N-1}\end{array}\right]=\left[\begin{array}{l}x_{1}+d_{c} \\ x_{2}+2 d_{c} \\ \vdots \\ x_{N-2}+(N-1) d_{c}\end{array}\right]=X+\left[\begin{array}{c}d_{c} \\ 2 d_{c} \\ \vdots \\ (N-1) d_{c}\end{array}\right]=X+D_{C}$

For a linear sparse array with $L=9 \lambda \quad, \quad N=10 \quad, \quad d_{c}=\lambda / 2$, $L Z=9 \lambda / 2, D_{c}=[\lambda / 2, \lambda, 3 \lambda / 2,2 \lambda, 5 \lambda / 2,3 \lambda, 7 \lambda / 2,4 \lambda, 9 \lambda / 2]$. We request the main lobe's width is $5^{\circ}$ and the highest side lobe level is $-25 d B$.

Different methods, GA and CDMEA, were investigated and compared with simulation solutions in order to assess the effectiveness and the flexibility of CDMEA. The experiment parameters of GA are: $p_{c}=0.6, p_{m}=0.1$. The experiment parameters of CDMEA are: $d f=0.5$, the subpopulation size is 18 , the temporary subpopulation is 12. In two algorithms, the evolutionary generation is 100 and the population size is 30 . The simulation results are as following:

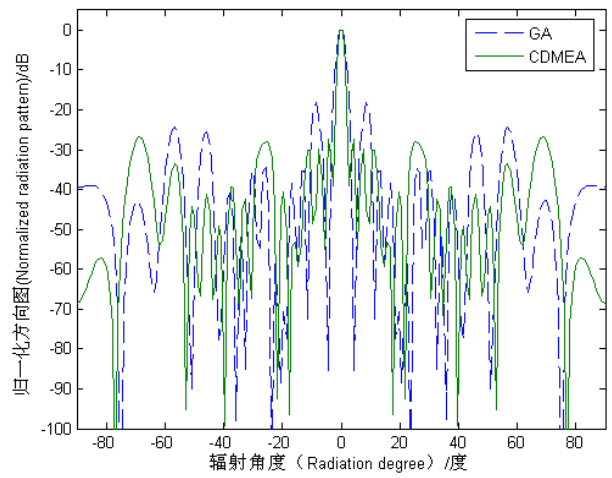

Figure 4. Optimization results of 10 elements sparse array for low SLL 
TABLE I. OPTIMIZATION RESULTS OF LINEAR SPARSE ARRAY ELEMENTS’ POSITIONS

\begin{tabular}{|c|c|c|c|c|}
\hline \multirow{2}{*}{$N$} & \multicolumn{2}{|c|}{ GA } & \multicolumn{2}{c|}{ CDMEA } \\
\cline { 2 - 5 } & $X$ & $D$ & $X$ & $D$ \\
\hline 1 & $0.2244 \lambda$ & $0.7244 \lambda$ & $0.3716 \lambda$ & $0.8716 \lambda$ \\
\hline 2 & $0.7831 \lambda$ & $1.7831 \lambda$ & $0.6793 \lambda$ & $1.6793 \lambda$ \\
\hline 3 & $0.8357 \lambda$ & $2.3357 \lambda$ & $1.2905 \lambda$ & $2.7905 \lambda$ \\
\hline 4 & $0.9060 \lambda$ & $2.9060 \lambda$ & $1.5816 \lambda$ & $3.5816 \lambda$ \\
\hline 5 & $0.9272 \lambda$ & $3.4272 \lambda$ & $3.0312 \lambda$ & $5.5312 \lambda$ \\
\hline 6 & $1.0026 \lambda$ & $4.0026 \lambda$ & $3.2885 \lambda$ & $6.2885 \lambda$ \\
\hline 7 & $1.0247 \lambda$ & $4.5247 \lambda$ & $3.7722 \lambda$ & $7.2722 \lambda$ \\
\hline 8 & $1.1175 \lambda$ & $5.1175 \lambda$ & $4.0185 \lambda$ & $8.0185 \lambda$ \\
\hline
\end{tabular}

From Fig.4, it can be seen that the highest side lobe level of GA is $-20 d B$, it does not satisfy the experiment request, while the highest side lobe level of DMEA reached $-25 d B$. From Table 1, we can see that the last element's position variable is $5.1173 \lambda$ in GA while the last position variable is $8.0183 \lambda$ in CDMEA when the solution space is $[0,8.5 \lambda]$. It shows the distribution optimized by GA does not give full play to the advantage of sparse antenna array. It is most likely to get into local optimum.

\section{Circular Sparse Antenna Array}

For a circular sparse antenna array with $R=2 \lambda, N=12, \varphi_{c}=\pi / 12, \theta_{0}=180^{0}$, then $L Z=2 \pi-12 \cdot(\pi / 12)=\pi$. We request the main lobe's width is $20^{\circ}$ and the highest side lobe level is $-20 d B$. The simulation results are as following:

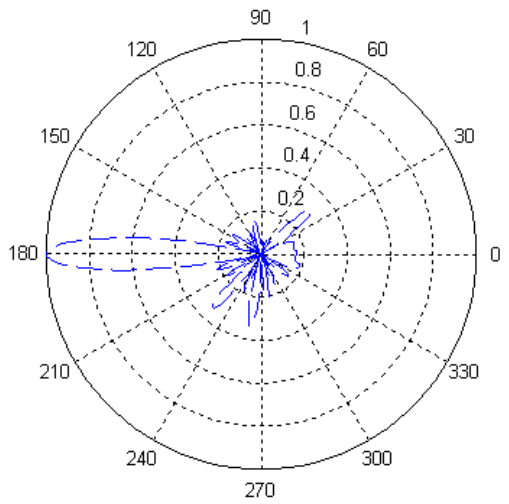

Figure 5. The polar pattern gained by CDMEA

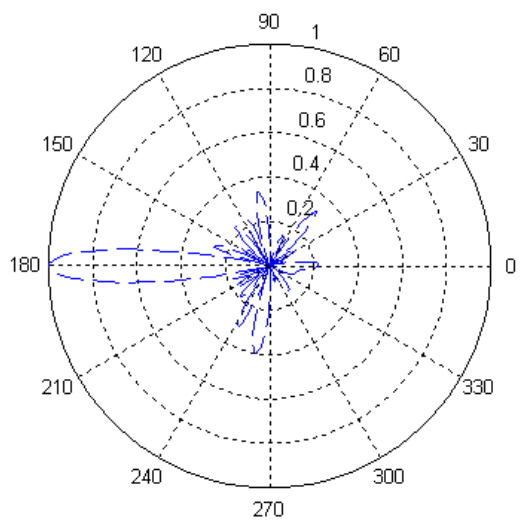

Figure 6. The polar pattern gained by GA 
TABLE II. OPTIMIZATION RESULTS OF CIRCULAR SPARSE ARRAY ELEMENTS’ POSITIONS

\begin{tabular}{|c|c|c|c|c|}
\hline \multirow{2}{*}{$\boldsymbol{N}$} & \multicolumn{2}{|c|}{$\mathrm{GA}$} & \multicolumn{2}{c|}{ CDMEA } \\
\cline { 2 - 5 } & $\boldsymbol{\psi}_{\boldsymbol{c}}$ & $\boldsymbol{\psi}$ & $\boldsymbol{\psi}_{\boldsymbol{c}}$ & $\boldsymbol{\psi}$ \\
\hline 1 & $5.20^{\circ}$ & $20.20^{\circ}$ & $0^{\circ}$ & $15.00^{\circ}$ \\
\hline 2 & $5.60^{\circ}$ & $35.60^{\circ}$ & $0^{\circ}$ & $30.00^{\circ}$ \\
\hline 3 & $9.17^{\circ}$ & $54.17^{\circ}$ & $2.00^{\circ}$ & $47.00^{\circ}$ \\
\hline 4 & $18.32^{\circ}$ & $78.32^{\circ}$ & $21.17^{\circ}$ & $81.17^{\circ}$ \\
\hline 5 & $28.35^{\circ}$ & $103.35^{\circ}$ & $26.83^{\circ}$ & $101.83^{\circ}$ \\
\hline 6 & $35.03^{\circ}$ & $125.03^{\circ}$ & $42.72^{\circ}$ & $132.72^{\circ}$ \\
\hline 7 & $35.04^{\circ}$ & $140.04^{\circ}$ & $53.63^{\circ}$ & $158.63^{\circ}$ \\
\hline 8 & $35.05^{\circ}$ & $155.05^{\circ}$ & $62.39^{\circ}$ & $182.39^{\circ}$ \\
\hline 9 & $35.07^{\circ}$ & $170.07^{\circ}$ & $166.97^{\circ}$ & $301.97^{\circ}$ \\
\hline 10 & $35.59^{\circ}$ & $185.59^{\circ}$ & $178.42^{\circ}$ & $328.42^{\circ}$ \\
\hline 11 & $43.68^{\circ}$ & $208.68^{\circ}$ & $180.00^{\circ}$ & $345.00^{\circ}$ \\
\hline
\end{tabular}

Fig.5 and Fig. 6 are the patterns of the circular sparse array in polar coordinate optimized by CDMEA and GA. We can see that circular array's scanning range reached to $360^{\circ}$ while the linear array's scanning range was only $180^{\circ}$. And there are only three side lobes whose normalized values exceeded 0.2 in Fig.5, while there are eight side lobes whose normalized values exceeded 0.2 in Fig.6. And like Table 1, Table 2 also shows the distribution optimized by GA does not give full play to the advantage of sparse antenna array.

\section{Conclusion}

Point at the defects of MEA - the generation of the initial population is blind and the addition of naturally washed out temporary subpopulations is random. We introduced improved Tent mapping and mutation operation of DEA into MEA and proposed CDMEA, which brought adequate diversity to the initial population and saved the excellent genes in the evolution. Then CDMEA is adopted to optimize the linear sparse array and circular sparse array. Computer simulations show that CDMEA can be applied in optimization problems of sparse antenna array and the optimization result is better than that obtained from GA.

\section{References}

[1] K. M. Xie, Y. G. Du and C. Y. Sun, "Application of the Mind-Evolution-Based Machine Learning in Mixture-Ratio Calculation of Raw Materials Cement", Proceedings of the 3rd World Congress on Intelligent Control and Automation, pp. 132-134, 2000.(in Chinese)

[2] K. Keiji, "Stability extended delayed-feedback control for discrete time chaotic systems", IEEE Trans. On Circuits and Systems, vol. 46, pp.1285-1288, Oct 1999.

[3] L. Chen, G. R. Chen, "Fuzzy modeling, prediction and control of uncertain chaotic systems based on time series", IEEE Trans. Circuits and Systems-I: Fundamental Theory and Application, vol. 47, pp.1527-1531, Oct 2000.

[4] R. L. Devaney, “An Introduction to Chaotic Dynamical Systems”, Addison-Wesley, 2nd ed, 1989, New York.

[5] R. Storn and K. Price, "Differential Evolution - A Simple and Efficient Adaptive Scheme for Global Optimization over Continuous Spaces”, Technical Report, International Computer Science Institute, vol. 8, pp. 22-25, Aug 1995.

[6] K. S. Chen, C. L. Han and Z. S. He, "A Synthesis Technique for Linear Sparse Arrays with Optimization Constraint of Minimum Element Spacing”, Chinese Journal of Radio Science, vol. 22, pp. 27-32, Feb 2007. (in Chinese) 
[7] B. P. Kumar and G. R. Branner, "Design of Unequally Spaced Arrays for Performance Improvement", IEEE Trans. on Antenna and Propagation., vol. 3, pp. 511-523, 1999.

[8] E. M. Thomas and M. P. Krishma, "Pattern Synthesis of Conformal Arrays for Airborne Vehicles", IEEE Aerospace Conference Proceeding, vol. 2, pp. 1030-1038, 2004.

[9] G. Caille, E. Vourch and M. J.Martin, "Conformal Array Antenna for Observation platforms in low Earth Orbit”, IEEE Antenna and Propagation Magazine, vol. 44, pp. 103-104,2002.

[10] V. Murino, A. Trucco and C. S. Regazzoni, "Synthesis of Unequally Spaced Arrays by Simulated Annealing", IEEE Trans. Antennas Signal Processing, vol. 44. pp. 119-123, 1996.

[11] K. S. Chen, Z. S. He and C. L. Han, "Design of Unequally Spaced Arrays for Performance Improvement", Sensor Array and Multichannel Signal Processing, IEEE Workshop 2006, pp. 166-170, 2006. (in Chinese) 